OPEN ACCESS

Edited by:

Raquel Largo,

Health Research Institute Foundation

Jimenez Diaz (IIS-FJD), Spain

Reviewed by:

Yuanli Chen,

Hefei University of Technology, China

Ting Zheng,

Qilu University of Technology, China

Mervyn Weitzmann,

Emory University, United States

${ }^{*}$ Correspondence:

Xuefeng Zhou

xfzhou@scsio.ac.cn

Gang Huang

huanggang@smu.edu.cn

Xiaojuan $\mathrm{Li}$

lixiaoj@smu.edu.cn

${ }^{\dagger}$ These authors have contributed equally to this work and share first authorship

Specialty section: This article was submitted to Inflammation Pharmacology,

a section of the journal

Frontiers in Pharmacology

Received: 04 August 2021 Accepted: 19 November 2021

Published: 17 January 2022

Citation:

Tan Y, Ke M, Li Z, Chen Y, Zheng J, Wang $Y$, Zhou $X$, Huang $G$ and $L i X$

(2022) A Nitrobenzoyl Sesquiterpenoid

Insulicolide A Prevents Osteoclast

Formation via Suppressing c-Fos-

NFATC1 Signaling Pathway.

Front. Pharmacol. 12:753240.

doi: 10.3389/fphar.2021.753240

\section{A Nitrobenzoyl Sesquiterpenoid Insulicolide A Prevents Osteoclast Formation via Suppressing c-Fos-NFATc1 Signaling Pathway}

\author{
Yanhui Tan ${ }^{1,2 \dagger}$, Minhong $\mathrm{Ke}^{2 \dagger}$, Zhichao $\mathrm{Li}^{1}$, Yan Chen ${ }^{2}$, Jiehuang Zheng ${ }^{2}$, Yiyuan Wang $^{2}$, \\ Xuefeng $\mathrm{Zhou}^{3 *}$, Gang Huang ${ }^{4 *}$ and Xiaojuan $\mathrm{Li}^{2 *}$ \\ ${ }^{1}$ State Key Laboratory for Chemistry and Molecular Engineering of Medicinal Resources, Collaborative Innovation Center for \\ Guangxi Ethnic Medicine, School of Chemistry and Pharmaceutical Sciences, Guangxi Normal University, Guilin, China, \\ ${ }^{2}$ Laboratory of Anti-inflammatory and Immunomodulatory Pharmacology, Guangdong Provincial Key Laboratory of New Drug \\ Screening, School of Pharmaceutical Sciences, Southern Medical University, Guangzhou, China, ${ }^{3}$ CAS Key Laboratory of \\ Tropical Marine Bio-resources and Ecology, Guangdong Key Laboratory of Marine Materia Medica, South China Sea Institute of \\ Oceanology, Chinese Academy of Sciences, Guangzhou, China, ${ }^{4}$ Integrated Traditional Chinese and Western Medicine Hospital, \\ Southern Medical University, Guangzhou, China
}

It is a viable strategy to inhibit osteoclast differentiation for the treatment of osteolytic diseases such as osteoporosis, rheumatoid arthritis and tumor bone metastases. Here we assessed the effects of insulicolide $A$, a natural nitrobenzoyl sesquiterpenoid derived from marine fungus, on receptor activator of nuclear factor- $\mathrm{kB}$ ligand (RANKL)-stimulated osteoclastogenesis in vitro and its protective effects on LPS-induced osteolysis mice model in vivo. The results demonstrated that insulicolide A inhibited osteoclastogenesis from $1 \mu \mathrm{M}$ in vitro. Insulicolide A could prevent c-Fos and nuclear factor of activated T-cell cytoplasmic 1 (NFATc1) nuclear translocation and attenuate the expression levels of osteoclast-related genes and DC-STAMP during RANKL-stimulated osteoclastogenesis but have no effects on NF-kB and MAPKs. Insulicolide A can also protect the mice from LPS-induced osteolysis. Our research provides the first evidence that insulicolide A may inhibit osteoclastogenesis both in vitro and in vivo, and indicates that it may have potential for the treatment of osteoclast-related diseases.

Keywords: insulicolide A, osteoclast, receptor activator of nuclear factor-kB ligand (RANKL), LPS, nuclear factor of activated T-cells cytoplasmic 1 (NFATc1)

\section{INTRODUCTION}

Bone resorption and formation are keeping a dynamic balance to maintain skeletal renewal and integrity. However, hyperactivity of osteoclast can lead to bone osteoclastic diseases such as osteoporosis, rheumatoid arthritis (RA), and tumor bone metastases (Perpetuo et al., 2017; Tsukasaki and Takayanagi, 2019; Györi and Mócsai, 2020; Kim et al., 2020; Liu et al., 2021). Therefore, targeting osteoclast formation has been regarded as a practicable treatment strategy to improve the prognosis of patients with bone destructive diseases (Broadhead et al., 2011; Stickeler and Fehm, 2014).

Osteoclasts, originate from bone marrow mononuclear macrophage lineage, are multinuclear and functioning as bone-resorbing cells (Boyle et al., 2003). Both receptor activator of nuclear factor- $\mathrm{kB}$ ligand (RANKL) and macrophage colony-stimulating factor (M-CSF) are essential for proliferation 
and differentiation of osteoclast (Arai et al., 1999; Cappellen et al., 2002; Lorenzo, 2017). Once RANKL is bound to its homologous receptor RANK, it first engages the adaptor protein tumor necrosis factor receptor-associated factor 6 (TRAF6) (Tanaka et al., 2005), then quickly triggers the signal cascade including nuclear factor NF- $\mathrm{kB}$, mitogen-activated protein kinases (MAPKs) (Matsumoto et al., 2000; Li et al., 2003; Wada et al., 2006; Lee et al., 2016), followed by activating c-Fos (Grigoriadis et al., 1994). Activated NF- $\kappa B$ or c-Fos can induce the activation and amplification of the downstream nuclear factor of activated T-cell cytoplasmic 1 (NFATc1), which can initiate the expression of osteoclast-related genes including OSCAR, Blimp1, DCSTAMP, cathepsin K, TRAP, and so on (Takayanagi et al., 2002; Edwards and Mundy, 2011).

Natural products from marine fungus have become a rich source for developing novel drugs for the treatment of various diseases (Kang et al., 2015; Malve, 2016). Nitrobenzoyl sesquiterpenoids (NSs) represent a novel and rare class of compounds isolated from marine fungi, and only seven are identified until now (Wu et al., 2012; Zhao et al., 2016; Tan et al., 2018). We have identified one NS compound, $6 \beta, 9 \alpha$ dihydroxy-14-p-nitrobenzoylcinnamolide (NS4), having the potential to suppress osteoclast formation by inhibiting NF$\kappa \mathrm{B} /$ RelB signaling pathway via binding to Arg B246 of NF- $\mathrm{\kappa B}$ P65 (Tan et al., 2020). Another NS compound, insulicolide A, isolated from the marine-derived fungus Aspergillus ochraceus, has demostrated antiinflammation and antitumor activity in vitro (Wang et al., 2014; Guo et al., 2018). However, the influence of insulicolide A on osteoclast differentiation in vitro and bone lysis in vivo is not yet known.

Here, our study evaluated the suppressive effect of insulicolide A on RANKL-stimulated bone marrow monocytes (BMMs)derived osteoclastogenesis in vitro, and tested the potential protective effects of insulicolide A on a LPS-induced osteolysis mice model in vivo. Insulicolide A mitigated osteoclastogenesis by preventing activation of c-Fos and NFATc1 but not affecting NF$\mathrm{\kappa B}$ signaling pathway.

\section{METHODS}

\section{Reagents and antibodies}

Insulicolide A was extracted from the cultured Aspergillus ochraceus Jcma1F17 derived from marine fungus according to the methods described previously (Tan et al., 2018). The structure of the compound was identified by both high-resolution mass spectrometry (HRMS) and nuclear magnetic resonance (NMR). The purity of the sample was more than $95 \%$ as analyzed by highperformance liquid chromatography (HPLC). The compound was kept under $-20^{\circ} \mathrm{C}$ for long-term storage and dissolved in dimethyl sulfoxide to a concentration of $10 \mathrm{mM}$ as a reserve before usage. Our previous study described the extracting craft and structure of insulicolide A (Wu et al., 2012; Zhao et al., 2016; Tan et al., 2018). RAW264.7 cells stably transfected with luciferase reporter genes of NF- $\mathrm{kB}$ and NFATc1 is a gift from Professor $\mathrm{Xu}$ (University of Western Australia, Nedlands,
Australia). Dulbecco's modified Eagle's medium (DMEM) and alpha minimum essential medium ( $\alpha$-MEM) were provided by Gibco (Rockville, MD, USA). Recombinant mouse M-CSF and RANKL are both from R\&D (Minneapolis, Minnesota, USA). MTT, TRAP assay Kit, BAY11-7082, and CSA were all provided by Sigma- Aldrich (St. Louis, MO, USA). Osteo Assay Surface Polystyrene Microplates were provided by Corning (St. Lowell, MA, USA). qPCR Master Mix were obtained from Promega (United States). RNeasy kit and PrimeScript RT reagent kit were purchased from TaKaRa (China). Nuclear isolation kit was obtained from Cayman Chemicals (Ann Arbor, MI, USA). Rabbit mAbs of p65 (\#49445), NFATc1 (\#8032), p-ERK (\#4370), ERK (\#4695), p-p38 (\#9215), p38 (\#9255), p-JNK (\#9255), JNK (\#9215), c-Fos (\#2250), $\beta$-actin (\#3700), and murine mAb of lamin A/C (\#4777) were all provided by CST (Beverly, MA, USA).

\section{Mice}

Mice of C57BL/6J and ICR were provided by the medical animal center in Guangdong province, China. The mice were housed in an environment with the temperature of $22-24^{\circ} \mathrm{C}$, a light/dark cycle of $12 \mathrm{~h}$ and $50-55 \%$ humidity. Water and food were provided at liberty. Animal studies were approved by the Committee of the animal protection and utilization of Southern Medical University and institutional animal protection and utilization of Guangxi Normal University.

\section{Cell culture}

The marrow cavity of C57BL/6J mice with 6-8 weeks of age was exposed and flushed with sterile PBS under sterile conditions. Cells were then collected, and red blood cells were lysed accordingly. The obtained cells were incubated in $\alpha$-MEM medium including $10 \%$ fetal bovine serum (FBS), $1 \%$ penicillin/streptomycin, and $50 \mathrm{ng} / \mathrm{ml}$ of M-CSF, and then nonadherent cells were collected for subsequent use. The mouse RAW264.7 cells transfected with luciferase reporter gene of NFATc1 were incubated in complete DMEM with $10 \%$ FBS in an incubator with $5 \% \mathrm{CO}_{2}$ at $37^{\circ} \mathrm{C}$.

\section{Cell viability assay}

BMMs $\left(1 \times 10^{3}\right.$ cells/well $)$ with or without insulicolide A were treated in $\alpha$-MEM medium supplemented with $50 \mathrm{ng} / \mathrm{ml}$ of M-CSF for 4 days. MTT assay was used to test cell viability following the instructions of the manufacturer.

\section{Osteoclastogenesis and tartrate-resistant acidic phosphatase assay}

For osteoclastogenesis assay, BMMs $\left(1 \times 10^{4}\right.$ cells/well $)$ were first incubated with different levels of insulicolide $\mathrm{A}$ in a 96well plate, then stimulated with $100 \mathrm{ng} / \mathrm{ml}$ of RANKL or $100 \mathrm{ng} /$ $\mathrm{ml}$ of LPS and $50 \mathrm{ng} / \mathrm{ml}$ of M-CSF for 3 days. After that, the cells were fixed in $2.5 \%$ glutaraldehyde and then stained to assay tartrate-resistant acidic phosphatase (TRAP) activity. Images were taken, and quantitation of osteoclasts (nuclei $>5$ for BMMs) were counted. 


\section{Bone resorption pit assay}

BMMs $\left(1 \times 10^{4}\right.$ cells/well $)$ were first incubated in Osteo Assay Surface Polystyrene Microplate, then administered with insulicolide $\mathrm{A}$ at different concentrations, stimulated by $50 \mathrm{ng} / \mathrm{ml}$ of M-CSF and $100 \mathrm{ng} / \mathrm{ml}$ of RANKL for 7 days. After that, $10 \%$ bleach solution was used to wash the cells. The resorption areas of osteoclast were quantified by ImagePro Plus 6.0.

\section{Nuclear factor of activated T-cell cytoplasmic 1 luciferase reporter assay}

The activity of luciferase reporter gene of NFATc1 induced by RANKL was measured as mentioned earlier. RAW264.7 cells, which were transfected with a NFATc1-responsive luciferase construct, were pretreated with insulicolide $\mathrm{A}$ and CsA (NFATc1 inhibitor, $1 \mu \mathrm{M}$ ) for $4 \mathrm{~h}$. After incubated by $100 \mathrm{ng} /$ $\mathrm{ml}$ of RANKL for $12 \mathrm{~h}$, the activity of luciferase was assayed.

\section{Quantitative real-time polymerase chain reaction}

Briefly, BMMs $\left(1 \times 10^{6}\right.$ cells $\left./ \mathrm{ml}\right)$ were treated by insulicolide A at different levels for $4 \mathrm{~h}$, followed by stimulation by RANKL and $\mathrm{M}$-CSF for $24 \mathrm{~h}$. The RNeasy mini kit was first used to isolate the total RNA, then, PrimeScript RT kit was prepared for synthesis of cDNA. Real-time PCR was performed using qPCR Master Mix. Polymerase chain reaction was executed by a procedure of $95^{\circ} \mathrm{C}$ $(30 \mathrm{~s}), 95^{\circ} \mathrm{C}(5 \mathrm{~s})$, and $60^{\circ} \mathrm{C} \mathrm{(34} \mathrm{s)} \mathrm{in} 40$ cycles. The mouse primers used are as follows: DC-STAMP (forward: AGACGTGGTTTA GGAATGCAGCTC; reverse: TCCTCCATGAACAAACAGTTC CAA), cathepsin K (forward: GGCCAACTCAAGAAGAAAAC; reverse: GTGCTTGCTTCCCTTCTGG), GAPDH (forward: ACACATTGGGGGTAGGAACA; reverse: AACTTTGGCATT GTGGAAGG), OSCAR (forward: CCTAGCCTCATACCC CCAG; reverse: CGTTGATCCCAGGAGTCACAA). The comparative $2^{-\triangle \Delta C T}$ method was used to calculate the relative expression of target genes. The mean $\mathrm{Ct}$ value of target genes in the experimental groups were normalized to the $\mathrm{Ct}$ values of GAPDH.

\section{Western blot analysis}

BMMs $\left(1 \times 10^{6}\right.$ cells $\left./ \mathrm{ml}\right)$ were treated with different concentrations of Insulicolide A for $4 \mathrm{~h}$, then incubated with $100 \mathrm{ng} / \mathrm{ml} \mathrm{RANKL}$ for additional $30 \mathrm{~min}$ or $24 \mathrm{~h}$. Total proteins were extracted by using RIPA buffer and cytoplasmic and nuclear proteins were prepared with a nuclear extraction kit. Proteins were separated on SDS-PAGE, and then transferred to PVDF membranes. The primary antibodies were used to incubate with membranes at $4^{\circ} \mathrm{C}$ overnight after blocked by $5 \%$ non-fat milk for $1 \mathrm{~h}$. On the next day, TBST was used to wash the membranes and then the secondary antibodies were used to incubate for another $1 \mathrm{~h}$ at room temperature. Finally, the blotted protein bands were obtained with a chemilunimescence kit (Yeasen Biotech, China) and quantification of the band intensities was analyzed by ImageJ software. The expression of p65, p38, ERK, JNK, p-ERK, p-p38, $\mathrm{p}$-JNK were measured after treatment of RANKL for $30 \mathrm{~min}$ and the expression of c-Fos, NFATc1, DC-STAMP were measured after RANKL treatment for $24 \mathrm{~h}$.

\section{LPS-induced murine inflammatory osteolysis model in vivo}

Female ICR mice aged 8-9 weeks were randomly divided into four groups with six mice in each group: the control group (served with PBS), model group (served with LPS), model with low dose Insulicolide A $(5 \mathrm{mg} / \mathrm{kg})$, and model with high dose Insulicolide A $(10 \mathrm{mg} / \mathrm{kg})$. LPS administration was by intraperitoneal injection ( $5 \mathrm{mg} / \mathrm{kg}$ body weight) on days 1 and 4 . Insulicolide A or PBS was administered once daily via gavage for 8 days. Eight days later, the ICR mice were euthanized. The left femur of all the animals were obtained and scanned by a micro-CT (CT80, ScancoMedical, Zurich, Switzerland) with the following instrument parameters: $50 \mathrm{kV}, 500 \mu \mathrm{A}$, and $0.7^{\circ}$ rotation step. The parameters of trabecular bone contains the ratio of bone volume to tissue volume (BV/TV), trabecular number (Tb.N), Mean density of TV and trabecular separation (Tb.Sp). Removal of the right femur from experimental mice to fix in $4 \% \mathrm{PFA}$ at $4^{\circ} \mathrm{C}$ for $24 \mathrm{~h}$, and then the femurs embedded in paraffin after decalcification in 12\% EDTA for 1 month were sectioned for H\&E and TRAP. As for the H\&E and TRAP staining images, there were $n=12$ images taken in total per group (two images from each mouse). A $600-\mu \mathrm{m} \times 600-\mu \mathrm{m}$ region of interest located $150 \mu \mathrm{m}$ below the growth plate of the femur metaphysis was employed for the assessment of the number of TRAP-positive multinucleated cells and osteoclastic surface/bone surface (Oc.S/ BS). Histomorphometry analysis was performed using Image Pro Plus 6.0 (IPP) software.

\section{Data analysis}

All data were expressed as the mean \pm standard deviation of three or more experiments. For multiple comparisons, differences were tested using a regular one-way ANOVA, followed by a Tukey multiple comparison for groups with a Gaussian distribution or with a Friedman test, followed by a Dunn's posttest for multiplicity if a Gaussian distribution could not be assumed. The $p$-values of less than 0.05 were deemed to be statistically significant.

\section{RESULTS}

\section{Insulicolide A inhibited receptor activator of nuclear factor- $\mathrm{kB}$ ligand-induced osteoclastogenesis in bone marrow monocytes in vitro}

To detect the effects of insulicolide A (Figure 1A) on RANKLinduced osteoclastogenesis, BMMs were first incubated with different concentrations of insulicolide A from 0.5 to $2 \mu \mathrm{M}$, then followed by incubation of RANKL and M-CSF. BMMs can differentiate into TRAP-positive osteoclasts in the presence of RANKL (Figures 1C, E). However, insulicolide A remarkably reduced osteoclastogenesis induced by RANKL 
A<smiles>C[C@]12CCC[C@@H](CO)[C@H]1[C@@H](OC(=O)c1ccc([N+](=O)[O-])cc1)C=C1C(=O)OC[C@]12O</smiles>

B

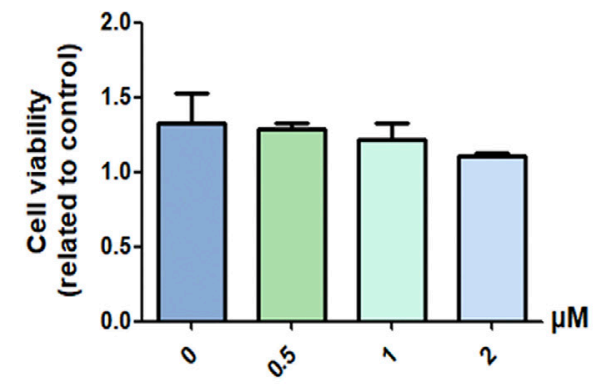

C

0

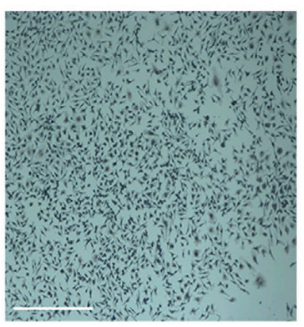

$$
0
$$

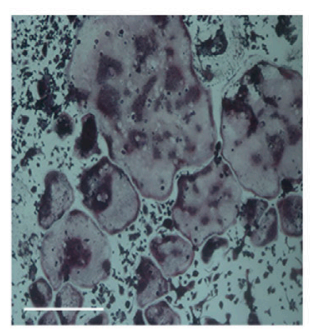

RANKL

0.5

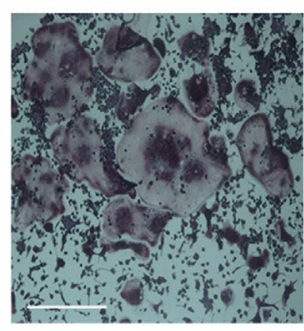

1

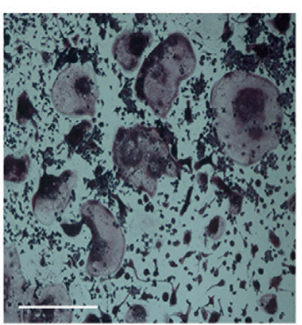

2 Insulicolide $A(\mu M)$

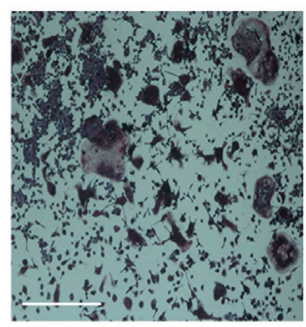

D

0

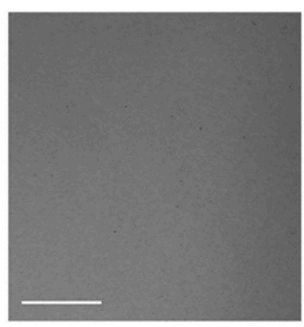

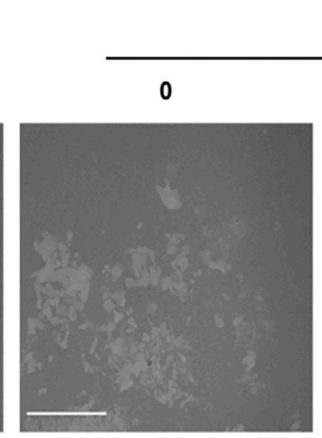

E

RANKL

0.5

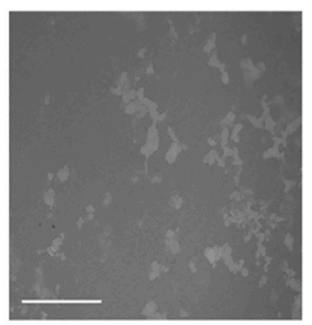

1

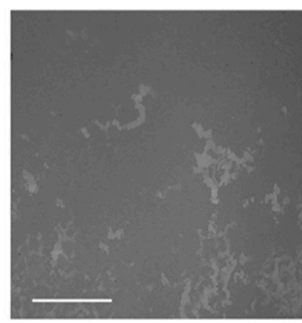

2 Insulicolide A ( $\mu \mathrm{M})$

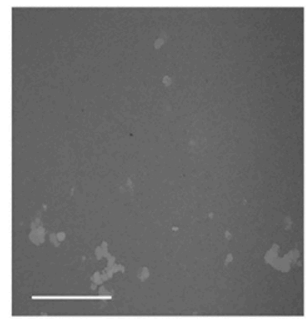

$\mathbf{F}$

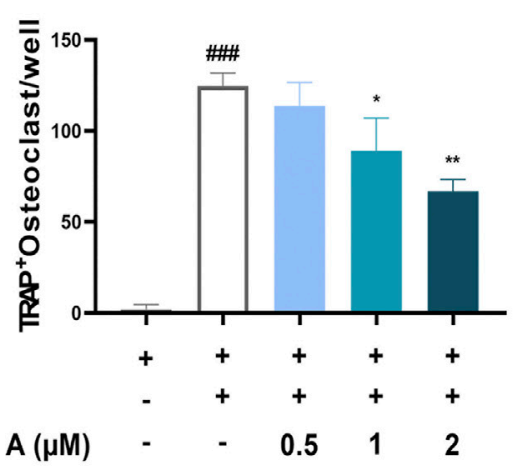

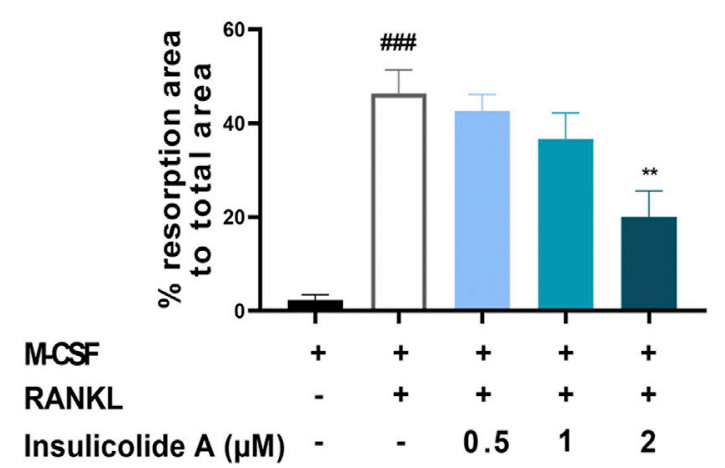

FIGURE 1 | Insulicolide A inhibited receptor activator of nuclear factor-kB ligand (RANKL)-induced osteoclastogenesis in vitro. (A) The chemical structure of insulicolide A. (B) The cell viability of 0.5-2 $\mu \mathrm{M}$ insulicolide A in bone marrow monocytes (BMMs) for 4 days were measured. (C, E) Images and number of TRAP-positive multinucleated cells (nuclei > 5) were taken and calculated. (D, F) Images and areas of bone resorption by osteoclasts on the hydroxyapatite-coated surfaces were taken and quantified. The data are shown as means \pm SD $(n=3$ independent experiments, containing three replicate samples each). \#\#\# $p<0.001$ vs. nontreatment groups, ${ }^{*} p<0.05,{ }^{* *} p<0.01$ vs. RANKL-induced groups. 
A

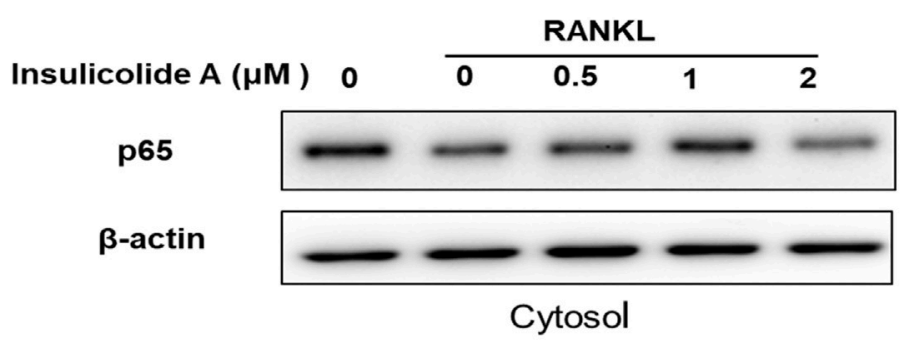

C

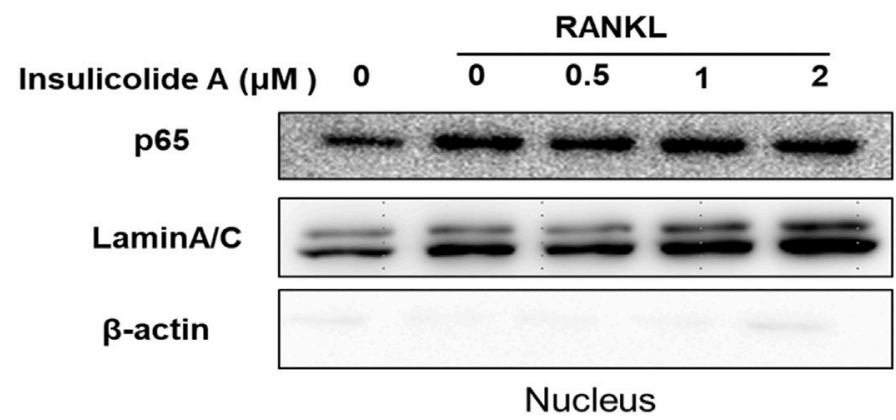

E

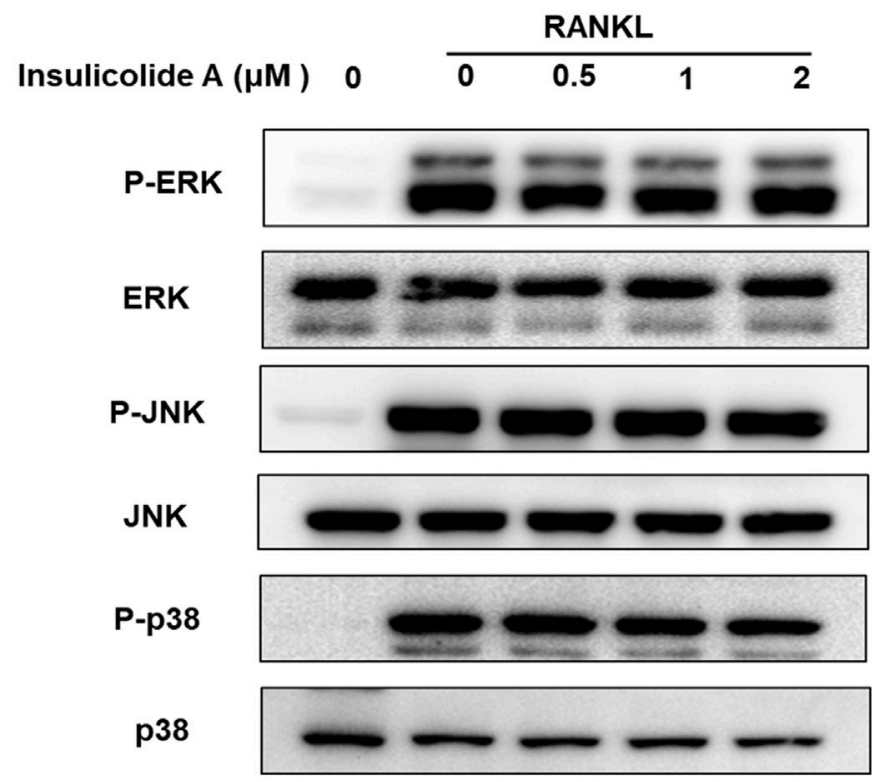

B
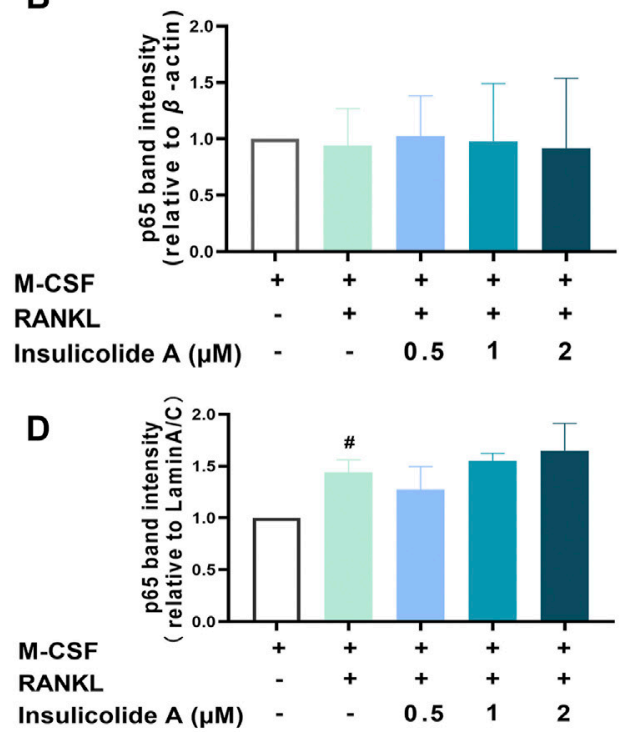

F

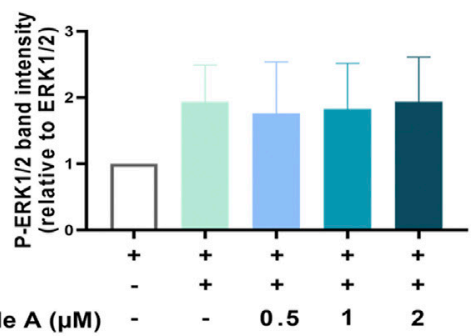

G

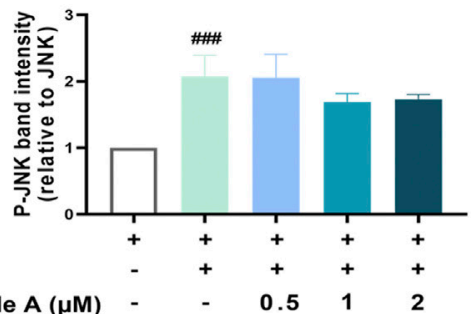

H

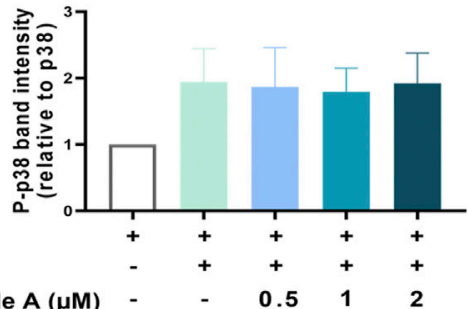

FIGURE 2 | Insulicolide A had little influence on activation of RANKL-induced NF- $\kappa B$ and mitogen-activated protein kinases (MAPKs). Insulicolide A (0.5-2 $\mu$ M) in BMMs were first cultured for $4 \mathrm{~h}$, then followed by $100 \mathrm{ng} / \mathrm{ml}$ of RANKL stimulation for $30 \mathrm{~min}$. After that, the proteins were harvested and detected by p65, LaminA/C, $\beta$-actin, ERK, P-ERK, JNK, P-JNK, p38, and P-p38 antibodies. The expression of nuclear or total protein levels of p65 (A, B) to $\beta$-actin, or p65 (C, D) to LaminA/C, and P-ERK to ERK, P-JNK to JNK or P-p38 to p38 (E-H) were determined by ImageJ software. The data are shown as means \pm SD of three independent experiments. $\# p<0.05,{ }^{\# \# \#} p<0.001$ vs. nontreatment groups.

in a dose-dependent manner without cytotoxicity (Figures 1B- C, E). Additionally, we found that insulicolide A also decreased LPS-induced osteoclastogenesis (Supplementary Figures S1A, B). Bone erosion is caused by the increased number of bone-resorbing osteoclasts, so we next evaluated the influence of insulicolide A on bone erosion of BMMs on hydroxyapatite-coated plates. Similar results were obtained, that reduced erosion areas was consistent with the reduced 


\section{A}

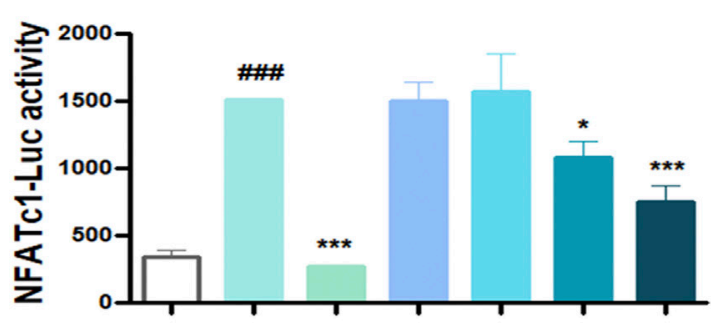

\section{M-CSF \\ RANKL Insulicolide A $(\mu M)$ CsA ( $\mu M)$}

$\begin{array}{rrrrrrr}+ & + & + & + & + & + & + \\ - & + & + & + & + & + & + \\ - & - & - & 0.5 & 1 & 2 & 4 \\ - & - & 1 & - & - & - & -\end{array}$

B

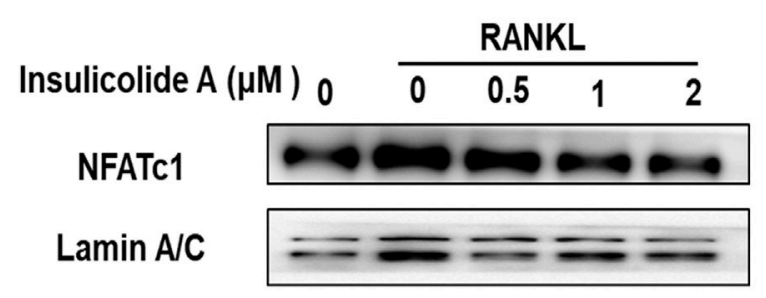

D

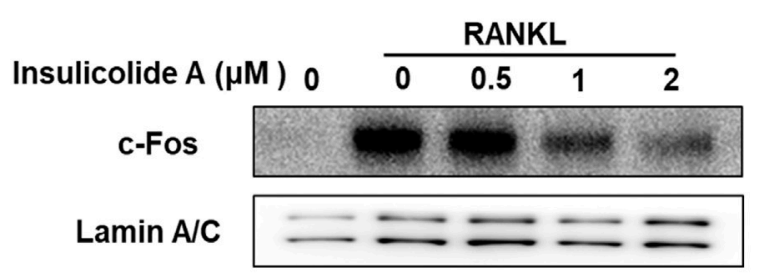

C

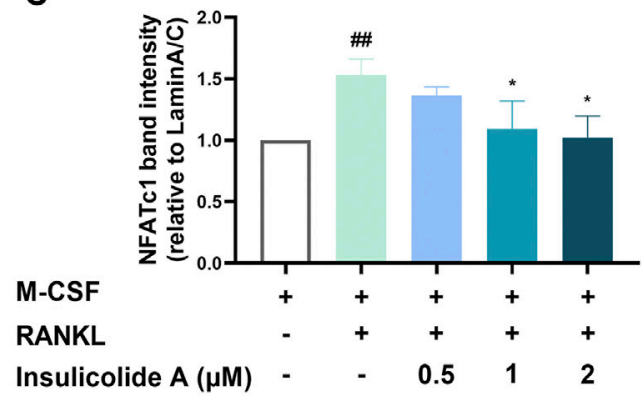

E

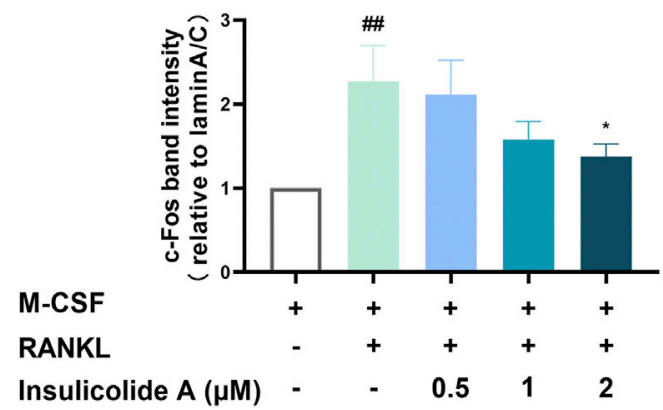

FIGURE 3 | Insulicolide A inhibited RANKL-induced c-Fos/nuclear factor of activated T-cells cytoplasmic 1 (NFATc1) signaling pathway. RAW264.7 cells, stably transfected by NFATc1 luciferase reporter vector, were first pretreated by insulicolide A $(0.5-4 \mu \mathrm{M})$ for $4 \mathrm{~h}$, then followed by $100 \mathrm{ng} / \mathrm{ml}$ of RANKL stimulation for $6 \mathrm{~h}$, and the luciferase activity (A) was assayed later. Insulicolide A (0.5-2 $\mu \mathrm{M})$ in BMMs were first cultured for $4 \mathrm{~h}$, then followed by $100 \mathrm{ng} / \mathrm{ml}$ of RANKL stimulation for 30 min. After that, nuclear proteins were harvested and detected by NFATc1, c-Fos, and LaminA/C antibodies. The relative expression of (B, C) NFATc1 to LaminA/C or (D,

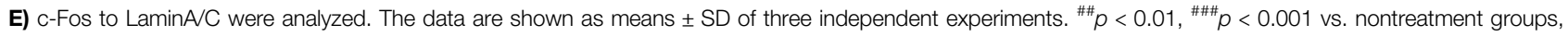
${ }^{\star} p<0.05,{ }^{\star \star \star} p<0.001$ vs. RANKL-induced groups.

osteoclast number by insulicolide $\mathrm{A}$, and the erosion areas were almost completely decreased by insulicolide A at $2 \mu \mathrm{M}$ (Figures 1D, F).

Insulicolide A had no statistically significant effects on activation of RANKL-induced NF- $\kappa \mathrm{B}$ and MAPKs.

Since we found insulicolide A attenuated osteoclast formation, we next elucidated the mechanisms of insulicolide A during RANKLinduced osteoclastogenesis. As NF- $\kappa \mathrm{B}$ and MAPKs signaling pathways are important in RANKL-induced osteoclast formation, we first investigated the effects of insulicolide A on RANKL-induced NF- $\kappa B$ activation including the protein expressions of NF- $\kappa B$ p 65 in cytosol and nucleus. Here, Western blotting assays indicated that the nuclear protein expression of NF- $\kappa \mathrm{B}$ p 65 increased remarkably after RANKL stimulation; however, insulicolide A from 0.5 to $2 \mu \mathrm{M}$ showed no statistically significant effects on p65 nuclear translocation (Figures 2A-D).

Then, we determined whether insulicolide A could attenuate the activation of MAPKs containing ERK/P-ERK, p38/P-p38, and JNK/P-JNK during RANKL-induced osteoclastogenesis. The protein expressions of P-ERK, P-p38, and P-JNK were enhanced rapidly after stimulation with RANKL; however, insulicolide A had no statistically significant effect on the phosphorylation of ERK, p38, and JNK, which was consistent with the influence on NF- $\mathrm{kB}$ (Figures $\mathbf{2 E - H}$ ). The above results 

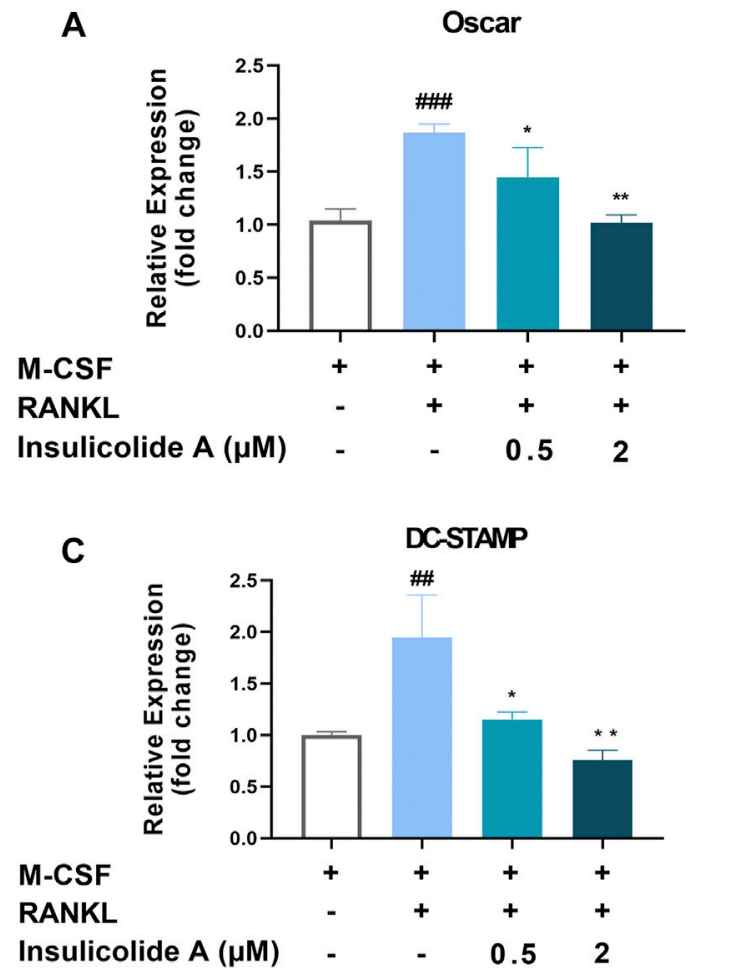

D

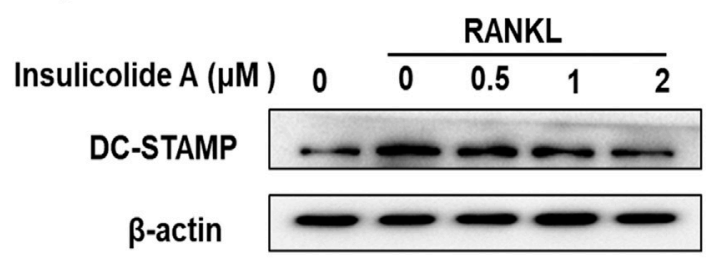

B

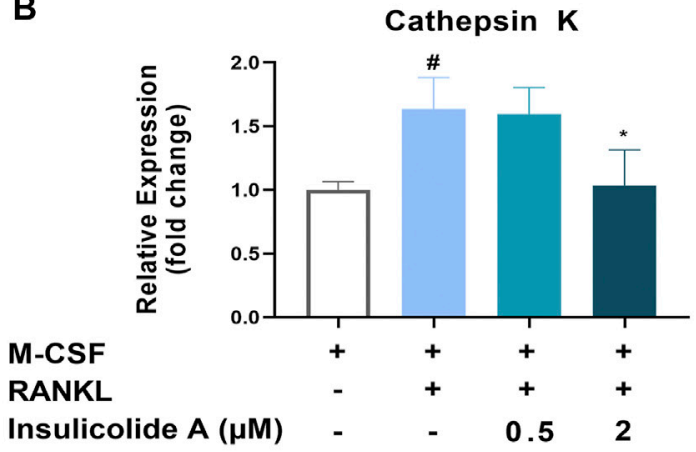

E

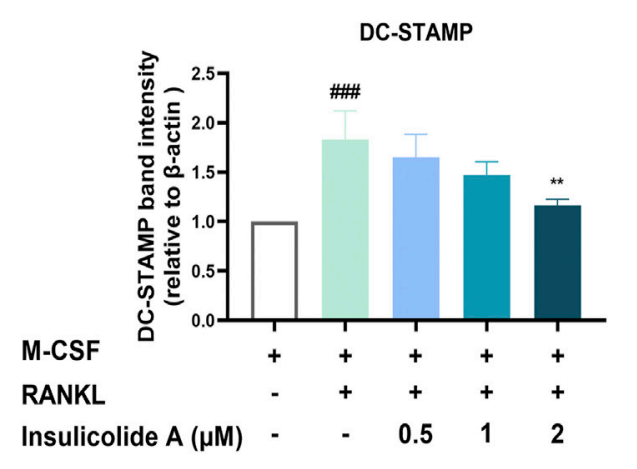

FIGURE 4 | Insulicolide A attenuated the expression of osteoclast-related genes and DC-STAMP induced by RANKL. Insulicolide A (0.5-2 $\mu M$ ) in BMMs were first cultured for $4 \mathrm{~h}$, then followed by $100 \mathrm{ng} / \mathrm{ml}$ of RANKL stimulation for $24 \mathrm{~h}$. After that, real-time PCR was used to analyz the gene expression of OSCAR (A), cathepsin $\mathrm{K}$ (B), and DC-STAMP (C). Antibodies were used to detect the total proteins expression of DC-STAMP and $\beta$-actin (D). (E) The relative expression of DC-STAMP to $\beta$-actin was analyzed by ImageJ software. The data are shown as means \pm SD of three independent experiments. ${ }^{\#} p<0.05$, ${ }^{\# \#} p<0.01$, ${ }^{\# \#} p<0.001$ vs. nontreatment groups, ${ }^{\star} p<0.05,{ }^{\star \star} p<0.01$ vs. RANKL-induced groups.

suggested that insulicolide A inhibits RANKL-induced osteoclast formation through other signaling pathways rather than NF- $\kappa B$ or MAPKs.

\section{Insulicolide A suppressed receptor activator of nuclear factor- $\mathrm{kB}$ ligand-induced $c$-Fos/nuclear factor of activated T-cell cytoplasmic 1 signaling pathway}

NFATc1, the master nuclear transcription factor, can activate osteoclastogenesis, and the activation of NFATc1 depends on NF$\kappa \mathrm{B}$, MAPKs signaling pathways, or its upstream transcriptional regulator c-Fos. Since insulicolide A has little effect on both RANKL-induced NF- $\kappa \mathrm{B}$ and MAPKs, we next examined the influence of insulicolide $\mathrm{A}$ on the activation of nuclear transcription factor c-Fos and NFATc1. First, we used NFATc1 luciferase reporter assay to determine the influence of insulicolide A on NFATc1 activation, and found that RANKLinduced NFATc1 luciferase activity was markedly suppressed by insulicolide $\mathrm{A}$ at 2 and $4 \mu \mathrm{M}$ concentrations (Figure 3A). Then Western blot assay revealed that the nucleus protein expression of RANKL-induced NFATc1 was increased. However, insulicolide A abrogated nucleus protein expression of NFATc1 in a dosedependent manner (Figures 3B, C). NFATcl self-amplification and activation are produced by the binding of up-stream nuclear transcription factor c-Fos, which combines with the NFATc1 promoter. C-Fos knockout mice showed severe osteosclerosis due to the reduced osteoclasts. In line with NFATc1, c-Fos protein levels were also restrained by insulicolide A (Figures 3D, E). 
A
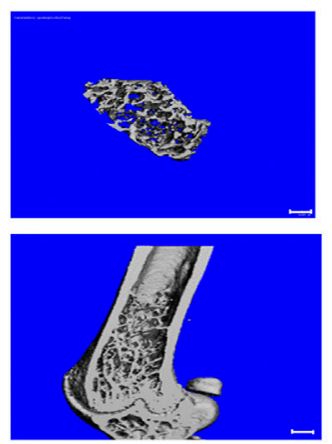

Control

B

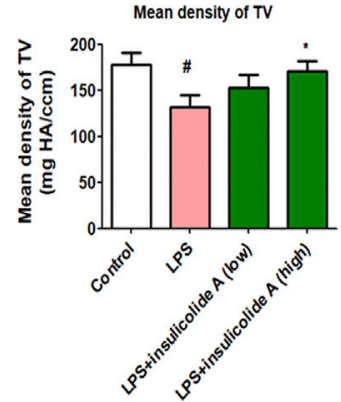

C

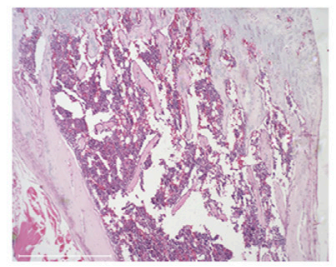

Control

D

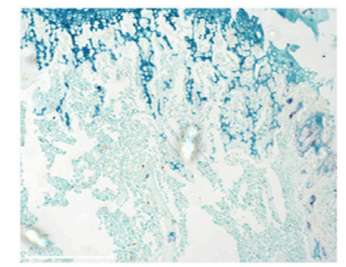

E

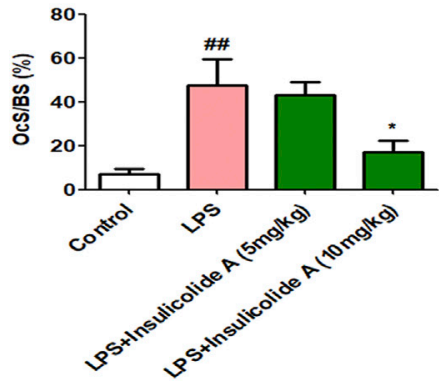

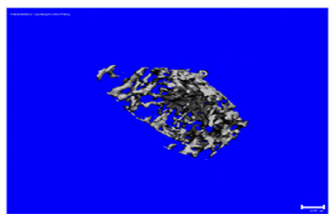
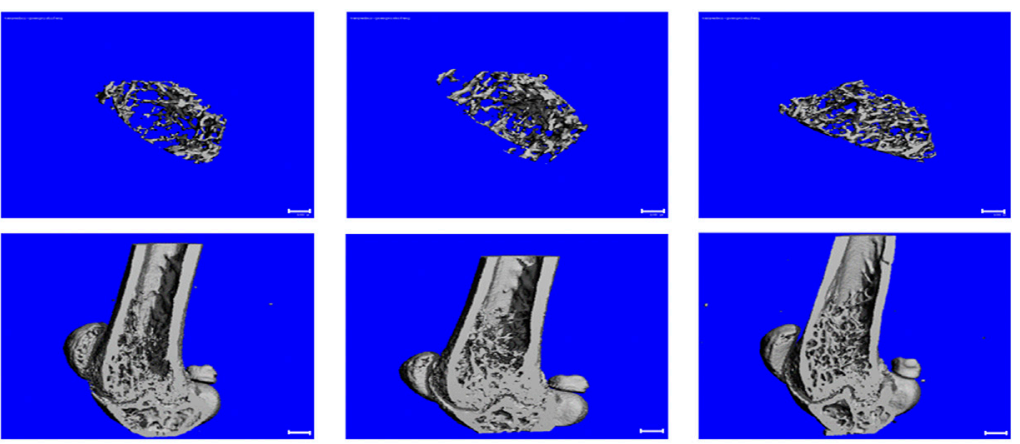

LPS

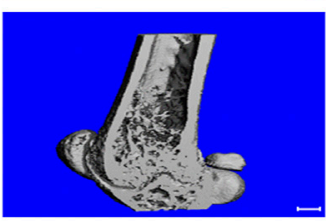

LPS+Insulicolide A (5mg/kg)

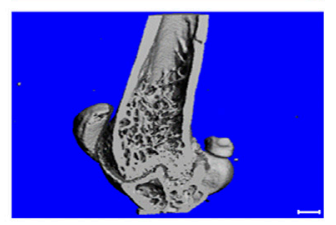

LPS+Insulicolide A (10 mg/kg)
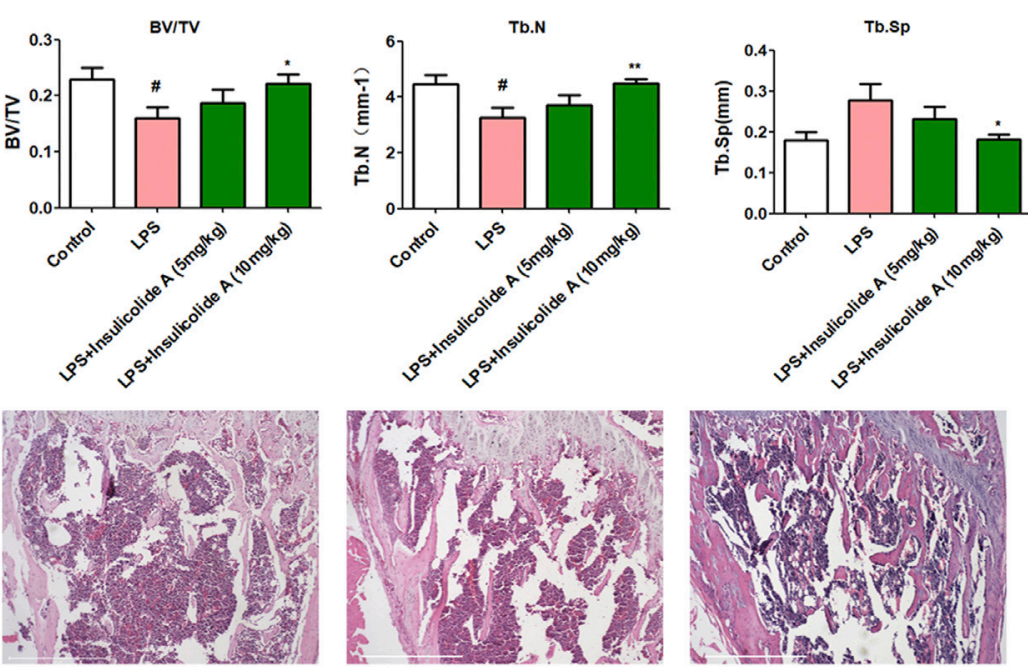

LPS

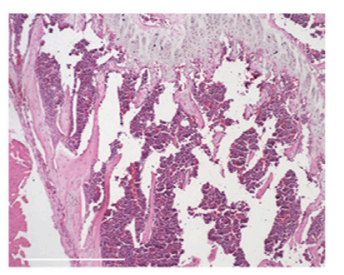

LPS+Insulicolide A $(5 \mathrm{mg} / \mathrm{kg})$
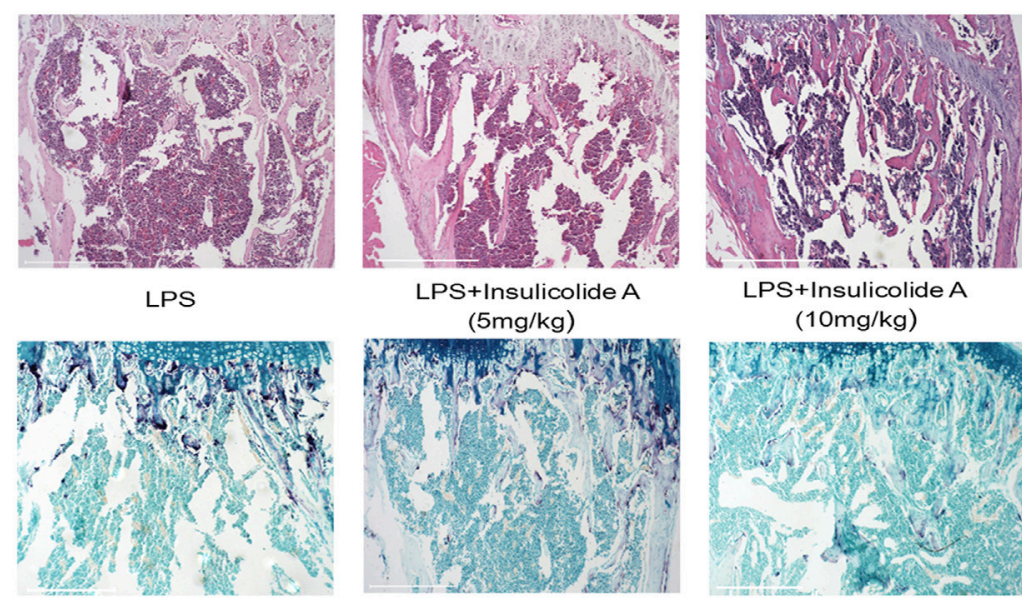

LPS+Insulicolide A $(10 \mathrm{mg} / \mathrm{kg})$

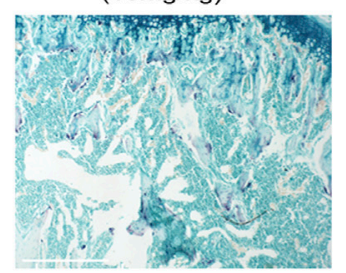

F

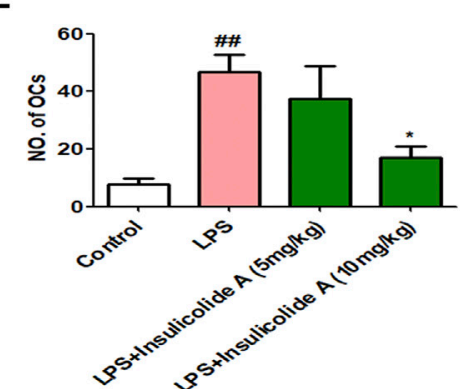

FIGURE 5 | Insulicolide A decreased LPS-induced murine bone loss by suppressing osteoclast activity. All mice were randomly divided into four groups with six mice in each group: control group (injected with PBS), LPS group (injected with LPS), LPS + insulicolide A (5 mg/kg) group, and LPS + insulicolide A (10 mg/kg) group. (A) Representative 3D reconstruction micro-CT images of transverse (above) and longitudinal (below) sections of the femur in different groups. (B) Quantitative measurements of trabecular bone parameters containing BMD, BV/TV, Tb.N, and Tb.Sp. Representative H\&E (C) and TRAP staining (D) images of the femurs in each group. Osteoclast surface/bone surface (E) and the number of osteoclasts in each group (F) were analyzed. $n=6$ mice per group. ${ }^{\#} p<0.05$, ${ }^{\# \#} p<0.01$ vs. PBStreatment groups, ${ }^{*} p<0.05,{ }^{\star *} p<0.01$ vs. LPS-treatment groups. 
Together, our results demonstrated that insulicolide A might attenuate RANKL-induced osteoclast formation by targeting the c-Fos/NFATc1 signaling pathway.

Insulicolide A attenuated the expression of osteoclast relative genes and DC-STAMP induced by RANKL.

Once NFATc1 is activated, osteoclast formation related genes, such as TRAP, OSCAR, cathepsin K, and osteoclast functionrelated genes including DC-STAMP, were strongly enhanced. However, when treated with insulicolide A, the mRNA expression of OSCAR, cathepsin K, and DC-STAMP were inhibited remarkably (Figures 4A-C). DC-STAMP, a multi-pass transmembrane molecule, is essential for the fusion and resorptive capacity of preosteoclasts. In DC-STAMP-deficient mice, the number of multinucleated osteoclasts reduced and bone mineral density increased. We then further examined the protein levels of DC-STAMP, consistent with the mRNA levels, insulicolide A also inhibited the protein levels of DC-STAMP induced by RANKL (Figures 4D, E). Collectively, these results suggested that insulicolide A inhibited RANKL-induced osteoclastogenesis and bone resorptive function by decreasing osteoclast formation-related genes and fusion-related genes.

\section{Insulicolide A decreased LPS-induced bone loss by inhibiting osteoclast activity}

LPS, an effective endotoxin from the cell wall in Gram-negative bacteria, can directly induce osteoblasts to secrete RANKL and then activate osteoclast formation. The activation of osteoclasts can result in osteoclastic diseases. In order to investigate the role of insulicolide $\mathrm{A}$ in the suppression of osteoclast function in vivo, LPS-induced inflammation bone loss mice model was used. MicroCT assays showed that LPS-injected mice model suffered a serious bone loss; however, when LPS-injected mice were treated with insulicolide A by gavage, both low-dose group ( $5 \mathrm{mg} / \mathrm{kg}$ ) and highdose group $(10 \mathrm{mg} / \mathrm{kg})$ could attenuate LPS-induced bone destruction (Figure 5A). Bone parameter analysis indicated that compared with LPS-injected mice, mean density of TV, BV/TV, Tb.N of insulicolide A $(10 \mathrm{mg} / \mathrm{kg})$-treated mice were markedly increased, but Tb.Sp was decreased (Figure 5B). H\&E-stained bone sections further confirmed the protection of insulicolide A on LPS-induced bone loss. Furthermore, TRAP staining assays and histomorphometric analysis of the number of osteoclasts and the percentage osteoclast surface per bone surface $(\mathrm{OcS} / \mathrm{BS})$ in the bone trabecula demonstrated that oral treatment of insulicolide A could remarkably decrease LPS-caused bone destruction and inhibited osteoclast numbers (Figures 5C-F). Taken together, our results indicated that oral administration of insulicolide $\mathrm{A}$ could prevent inflammatory bone destruction in vivo.

\section{DISCUSSION}

Sesquiterpenoids from the plants and microorganisms display various biological activities. Marine fungus-derived nitrobenzoyl sesquiterpenoids, rare from natural source, exhibit remarkable pharmacological activities, including antitumor and antiinflammation (Wang et al., 2014; Guo et al., 2018). Here our results indicated that marine-derived nitrobenzoyl sesquiterpenoid, insulicolide A, could attenuate osteoclastogenesis c-Fos-NFATc1 signaling pathway induced by RANKL in vitro at doses from 1 to $2 \mu \mathrm{M}$. Consistently, we also found that insulicolide A could protect inflammatory osteolysis in vivo.

During the process of RANKL-induced osteoclast formation, the combination of RANKL to RANK can recruit TRAF6 followed by activation of NF- $\kappa B$ and MAPKs signaling pathways. NF- $\kappa B$ and MAPKs are crucial pathways of RANKL response in osteoclastogenesis (Ghosh and Karin, 2002; Jimi and Ghosh, 2005). However, our data showed that insulicolide A had no significant reduction on $\mathrm{p} 65$ of NF- $\mathrm{KB}$ and the phosphorylation of ERK, p38, and JNK of MAPKs, which were incompletely consistent with the effects of NS4 (structural isomer of insulicolide A) on NF- $\kappa$ B. NS4 exhibited inhibitory effects on NF- $\kappa$ B by binding with NF- $\kappa$ B p65 Arg B246 (Tan et al., 2020), the different mechanisms between NS4 and insulicolide A on NF$\kappa \mathrm{B}$ may be due to their different conformations and their affinity with NF- $\kappa$ B. These results suggested that NF- $\kappa$ B and MAPKs might not be a downstream signal by insulicolide $\mathrm{A}$ to treat RANKL-induced osteoclast differentiation.

$\mathrm{C}-\mathrm{Fos}$, the subunit of activator protein-1 (AP-1), is induced during the process of RANKL-induced osteoclastogenesis (Grigoriadis et al., 1994). As the upstream nuclear transcription factor of NFATc1, activated c-Fos can bind to the promoter region of NFATc1 resulting in the autoamplification and activation of NFATc1 (Takatsuna et al., 2005). C-Fos knockout mice showed decreased NFATc1 nuclear translocation and severe bone sclerosis (Matsuo et al., 2004). Here, our findings determined that the nuclear protein level of c-Fos was drastically inhibited by insulicolide A in the BMMs of RANKL stimulation, which was also different with NS4, while NS4 had little effect on c-Fos nuclear expression during RANKL-induced osteoclast formation (Tan et al., 2020). Thus, the decrease in RANKL-induced c-Fos activation and the following attenuated NFATc1 nuclear protein levels, contributes to impaired osteoclastogenesis after insulicolide A treatment.

NFATc1 is the core transcriptional switch of osteoclast terminal differentiation (Nepal et al., 2013). Activated NFATc1 not only increases the expression levels of osteoclast-related genes including TRAP, OSCAR, and cathepsin $\mathrm{K}$, but also refers to the multinucleation of osteoclast precursors through cell fusion proteins such as DC-STAMP (Yagi et al., 2006; Chiu and Ritchlin, 2016). In our study, the levels of OSCAR and cathepsin $\mathrm{K}$ induced by RANKL were remarkably reduced after treatment of insulicolide A. Additionally, RANKL-induced mRNA and protein expressions of DC-STAMP in osteoclast formation were also down-regulated after treatment with insulicolide $\mathrm{A}$. The reduction of nuclear translocation of NFATc1 and the suppression of DC-STAMP caused by insulicolide A might attenuate the expression of osteoclast-specific genes and the fusion of preosteoclast cells to inhibit osteoclasts in vitro.

LPS is an effective endotoxin from the cell wall in Gramnegative bacteria, which has an important impact on bone loss (Place et al., 2021). In our experiment, we constructed murine bone loss model induced by LPS to evaluate the influence of insulicolide $\mathrm{A}$ on osteoclastogenesis in vivo. Micro-CT analysis indicated that oral insulicolide A $(10 \mathrm{mg} / \mathrm{kg})$ could recover bone 
loss induced by LPS through promoting BMD, BV/TV, and Tb.N, while reducing Tb.Sp. H\&E and TRAP-stained bone section analysis further showed that insulicolide A remarkably inhibited bone destruction and osteoclast numbers in LPS-induced mouse model. Therefore, these results further manifested that oral insulicolide A protected bone by decreasing the numbers of osteoclast and improving bone parameters in vivo, which were consistent with its inhibitory effects on osteoclasts in vitro.

The up-stream signals such as TRAF6, DC-STAMP, and NF$\mathrm{\kappa B} / \mathrm{MAPKs}$ regulate the expression of $\mathrm{c}$-Fos during osteoclastogenesis (Yin et al., 2017; Liu et al., 2021; Zou et al.,

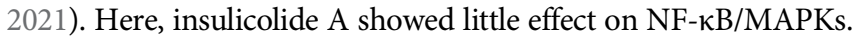
DC-STAMP knockdown decreases c-Fos and NFATc1 expression in osteoclast precursor cells (Yin et al., 2017), thus, DC-STAMP can also serve as the surface molecule for insulicolide A to suppress osteoclast formation. Furthermore, the analysis of proteins binding with insulicolide $\mathrm{A}$ in osteoclastogenesis can be performed to disclose the target of insulicolide $\mathrm{A}$ in the future. In addition, we found that insulicolide A protected bone only by resorption in vivo. Further studies aimed at bone formation in osteoclast-related mouse models may make a better understanding of this compound for its treatment of osteolytic diseases.

In conclusion, this study discovered that insulicolide $\mathrm{A}$, a natural nitrobenzoyl sesquiterpenoid isolated from the marinederived Aspergillus ochraceus fungus, can suppress RANKLinduced osteoclastogenesis by preventing c-Fos rather than NF- $\kappa \mathrm{B}$ and MAPKs, and then decreasing the level of NFATc1 and DC-STAMP in vitro. Our data, including the murine femur model experiment, suggest insulicolide $A$ as a substantial osteoclasts inhibitor and indicate its promising application for osteoclast overactivated diseases.

\section{DATA AVAILABILITY STATEMENT}

The original contributions presented in the study are included in the article/Supplementary Material, further inquiries can be directed to the corresponding authors.

\section{REFERENCES}

Arai, F., Miyamoto, T., Ohneda, O., Inada, T., Sudo, T., Brasel, K., et al. (1999). Commitment and Differentiation of Osteoclast Precursor Cells by the Sequential Expression of C-Fms and Receptor Activator of Nuclear Factor kappaB (RANK) Receptors. J. Exp. Med. 190 (12), 1741-1754. doi:10.1084/ jem.190.12.1741

Boyle, W. J., Simonet, W. S., and Lacey, D. L. (2003). Osteoclast Differentiation and Activation. Nature 423 (6937), 337-342. doi:10.1038/nature01658

Broadhead, M. L., Clark, J. C., Dass, C. R., Choong, P. F., and Myers, D. E. (2011). Therapeutic Targeting of Osteoclast Function and Pathways. Expert Opin. Ther. Targets 15 (2), 169-181. doi:10.1517/14728222.2011.546351

Cappellen, D., Luong-Nguyen, N. H., Bongiovanni, S., Grenet, O., Wanke, C., and Susa, M. (2002). Transcriptional Program of Mouse Osteoclast Differentiation Governed by the Macrophage colony-stimulating Factor and the Ligand for the Receptor Activator of NFkappa B. J. Biol. Chem. 277 (24), 21971-21982. doi:10.1074/jbc.M200434200

\section{ETHICS STATEMENT}

The study was reviewed and approved by the Committee of the animal protection and utilization of Southern Medical University and the institutional animal protection and utilization of Guangxi Normal University.

\section{AUTHOR CONTRIBUTIONS}

Study conception and design: XJL, YHT, and MHK. Acquisition, analysis, and interpretation of data: YHT, MHK, ZCL, YC, JHZ, and YYW. Drafting/revision of the work for intellectual content and context: YHT, MHK, XFZ, GH, and XJL. Final approval and overall responsibility for the published work: XFZ, GH and XJL.

\section{FUNDING}

This research was funded by the National Natural Science Foundation of China (81773740, U20A20101), the Natural Science Foundation of Guangxi (2021JJB140057), Project to improve the basic research ability of young and middle-aged university teachers of Guangxi (2021KY0062), Finance Science and Technology Project of Hainan Province (ZDKJ202018), Guangdong Local Innovation Team Program (2019BT02Y262), State Key Laboratory for Chemistry and Molecular Engineering of Medicinal Resources (CMEMR2019-A05), and Guangzhou Science and Technology Plan Project (201804010027).

\section{SUPPLEMENTARY MATERIAL}

The Supplementary Material for this article can be found online at: https://www.frontiersin.org/articles/10.3389/fphar.2021.753240/ full\#supplementary-material

Chiu, Y. H., and Ritchlin, C. T. (2016). DC-STAMP: A Key Regulator in Osteoclast Differentiation. J. Cel Physiol 231 (11), 2402-2407. doi:10.1002/jcp.25389

Edwards, J. R., and Mundy, G. R. (2011). Advances in Osteoclast Biology: Old Findings and New Insights from Mouse Models. Nat. Rev. Rheumatol. 7 (4), 235-243. doi:10.1038/nrrheum.2011.23

Ghosh, S., and Karin, M. (2002). Missing Pieces in the NF-kappaB Puzzle. Cell 109 Suppl (Suppl. 1), S81-S96. doi:10.1016/s0092-8674(02)00703-1

Grigoriadis, A. E., Wang, Z. Q., Cecchini, M. G., Hofstetter, W., Felix, R., Fleisch, H. A., et al. (1994). c-Fos: a Key Regulator of Osteoclast-Macrophage Lineage Determination and Bone Remodeling. Science 266 (5184), 443-448. doi:10.1126/science.7939685

Guo, L. M., Lyu, J. L., and Zhang, L. B. (2018). [Research Progress on Antiinflammatory Mechanism of Natural Sesquiterpenoids]. Zhongguo Zhong Yao Za Zhi 43 (20), 3989-3999. doi:10.19540/j.cnki.cjcmm.20180726.013

Győri, D. S., and Mócsai, A. (2020). Osteoclast Signal Transduction during Bone Metastasis Formation. Front Cel Dev Biol 8, 507. doi:10.3389/fcell.2020.00507

Jimi, E., and Ghosh, S. (2005). Role of Nuclear Factor-kappaB in the Immune System and Bone. Immunol. Rev. 208, 80-87. doi:10.1111/j.01052896.2005.00329.x 
Kang, H. K., Seo, C. H., and Park, Y. (2015). The Effects of marine Carbohydrates and Glycosylated Compounds on Human Health. Int. J. Mol. Sci. 16 (3), 6018-6056. doi:10.3390/ijms16036018

Kim, J. M., Lin, C., Stavre, Z., Greenblatt, M. B., and Shim, J. H. (2020). OsteoblastOsteoclast Communication and Bone Homeostasis. Cells 9 (9). doi:10.3390/ cells 9092073

Lee, K., Chung, Y. H., Ahn, H., Kim, H., Rho, J., and Jeong, D. (2016). Selective Regulation of MAPK Signaling Mediates RANKL-dependent Osteoclast Differentiation. Int. J. Biol. Sci. 12 (2), 235-245. doi:10.7150/ijbs.13814

Li, X., Udagawa, N., Takami, M., Sato, N., Kobayashi, Y., and Takahashi, N. (2003). p38 Mitogen-Activated Protein Kinase Is Crucially Involved in Osteoclast Differentiation but Not in Cytokine Production, Phagocytosis, or Dendritic Cell Differentiation of Bone Marrow Macrophages. Endocrinology 144 (11), 4999-5005. doi:10.1210/en.2003-0166

Liu, Y., Liu, W., Yu, Z., Zhang, Y., Li, Y., Xie, D., et al. (2021). A Novel BRD4 Inhibitor Suppresses Osteoclastogenesis and Ovariectomized Osteoporosis by Blocking RANKL-Mediated MAPK and NF-Kb Pathways. Cell Death Dis 12 (7), 654. doi:10.1038/s41419-021-03939-7

Lorenzo, J. (2017). The many Ways of Osteoclast Activation. J. Clin. Invest. 127 (7), 2530-2532. doi:10.1172/JCI94606

Malve, H. (2016). Exploring the Ocean for New Drug Developments: Marine Pharmacology. J. Pharm. Bioallied Sci. 8 (2), 83-91. doi:10.4103/0975-7406.171700

Matsumoto, M., Sudo, T., Saito, T., Osada, H., and Tsujimoto, M. (2000). Involvement of P38 Mitogen-Activated Protein Kinase Signaling Pathway in Osteoclastogenesis Mediated by Receptor Activator of NF-Kappa B Ligand (RANKL). J. Biol. Chem. 275 (40), 31155-31161. doi:10.1074/jbc.M001229200

Matsuo, K., Galson, D. L., Zhao, C., Peng, L., Laplace, C., Wang, K. Z., et al. (2004). Nuclear Factor of Activated T-Cells (NFAT) Rescues Osteoclastogenesis in Precursors Lacking C-Fos. J. Biol. Chem. 279 (25), 26475-26480. doi:10.1074/ jbc.M313973200

Nepal, M., Choi, H. J., Choi, B. Y., Yang, M. S., Chae, J. I., Li, L., et al. (2013). Hispidulin Attenuates Bone Resorption and Osteoclastogenesis via the RANKL-Induced NF-Kb and NFATc1 Pathways. Eur. J. Pharmacol. 715 (13), 96-104. doi:10.1016/j.ejphar.2013.06.002

Perpetuo, I. P., Caetano-Lopes, J., Rodrigues, A. M., Campanilho-Marques, R., Ponte, C., Canhao, H., et al. (2017). Effect of Tumor Necrosis Factor Inhibitor Therapy on Osteoclasts Precursors in Rheumatoid Arthritis. Biomed. Res. Int. 2017: 2690402. doi:10.1155/2017/2690402

Place, D. E., MalireddiSubbarao, R. K. S., Kim, J., Vogel, P., Yamamoto, M., and Kanneganti, T. D. (2021). Osteoclast Fusion and Bone Loss Are Restricted by Interferon Inducible Guanylate Binding Proteins. Nat. Commun. 12 (1), 496. doi:10.1038/s41467-020-20807-8

Stickeler, E., and Fehm, T. (2014). Targeted and Osteo-Oncologic Treatment in Early Breast Cancer: What Is State-Of-The-Art and what Might Become So within the Next 5 years? Breast Care (Basel) 9 (3), 161-167. doi:10.1159/ 000365129

Takatsuna, H., Asagiri, M., Kubota, T., Oka, K., Osada, T., Sugiyama, C., et al. (2005). Inhibition of RANKL-Induced Osteoclastogenesis by (-)-DHMEQ, a Novel NF-kappaB Inhibitor, through Downregulation of NFATc1. J. Bone Miner Res. 20 (4), 653-662. doi:10.1359/JBMR.041213

Takayanagi, H., Kim, S., Koga, T., Nishina, H., Isshiki, M., Yoshida, H., et al. (2002). Induction and Activation of the Transcription Factor NFATc1 (NFAT2) Integrate RANKL Signaling in Terminal Differentiation of Osteoclasts. Dev. Cel 3 (6), 889-901. doi:10.1016/s1534-5807(02)00369-6

Tan, Y., Deng, W., Zhang, Y., Ke, M., Zou, B., Luo, X., et al. (2020). A marine Fungus-Derived Nitrobenzoyl Sesquiterpenoid Suppresses Receptor Activator of NF-Kb Ligand-Induced Osteoclastogenesis and Inflammatory Bone Destruction. Br. J. Pharmacol. 177 (18), 4242-4260. doi:10.1111/bph.15179

Tan, Y., Yang, B., Lin, X., Luo, X., Pang, X., Tang, L., et al. (2018). Nitrobenzoyl Sesquiterpenoids with Cytotoxic Activities from a Marine-Derived Aspergillus ochraceus Fungus. J. Nat. Prod. 81 (1), 92-97. doi:10.1021/ acs.jnatprod.7b00698

Tanaka, S., Nakamura, K., Takahasi, N., and Suda, T. (2005). Role of RANKL in Physiological and Pathological Bone Resorption and Therapeutics Targeting the RANKL-RANK Signaling System. Immunol. Rev. 208, 30-49. doi:10.1111/ j.0105-2896.2005.00327.x

Tsukasaki, M., and Takayanagi, H. (2019). Osteoimmunology: Evolving Concepts in Bone-Immune Interactions in Health and Disease. Nat. Rev. Immunol. 19 (10), 626-642. doi:10.1038/s41577-019-0178-8

Wada, T., Nakashima, T., Hiroshi, N., and Penninger, J. M. (2006). RANKL-RANK Signaling in Osteoclastogenesis and Bone Disease. Trends Mol. Med. 12 (1), 17-25. doi:10.1016/j.molmed.2005.11.007

Wang, G. W., Qin, J. J., Cheng, X. R., Shen, Y. H., Shan, L., Jin, H. Z., et al. (2014). Inula Sesquiterpenoids: Structural Diversity, Cytotoxicity and Anti-tumor Activity. Expert Opin. Investig. Drugs 23 (3), 317-345. doi:10.1517/ 13543784.2014.868882

Wu, Q. X., Jin, X. J., Draskovic, M., Crews, M. S., Tenney, K., Valeriote, F. A., et al. (2012). Unraveling the Numerous Biosynthetic Products of the Marine Sediment-Derived Fungus, Aspergillus insulicola. Phytochem. Lett. 5 (1), 114-117. doi:10.1016/j.phytol.2011.11.005

Yagi, M., Miyamoto, T., Toyama, Y., and Suda, T. (2006). Role of DC-STAMP in Cellular Fusion of Osteoclasts and Macrophage Giant Cells. J. Bone Miner Metab. 24 (5), 355-358. doi:10.1007/s00774-006-0697-9

Yin, Y., Tang, L., Chen, J., and Lu, X. (2017). MiR-30a Attenuates Osteoclastogenesis via Targeting DC-STAMP-c-Fos-NFATc1 Signaling. Am. J. Transl Res. 9 (12), 5743-5753.

Zhao, H. Y., Anbuchezhian, R., Sun, W., Shao, C. L., Zhang, F. L., Yin, Y., et al. (2016). Cytotoxic Nitrobenzoyloxy-Substituted Sesquiterpenes from Spongederived Endozoic Fungus Aspergillus insulicola MD10-2. Curr. Pharm. Biotechnol. 17 (3), 271-274. doi:10.2174/1389201017666151223123424

Zou, B. H., Tan, Y. H., Deng, W. D., Zheng, J. H., Yang, Q., Ke, M. H., et al. (2021). Oridonin Ameliorates Inflammation-Induced Bone Loss in Mice via Suppressing DC-STAMP Expression. Acta Pharmacol. Sin 42 (5), 744-754. doi:10.1038/s41401-020-0477-4

Conflict of Interest: The authors declare that the research was conducted in the absence of any commercial or financial relationships that could be construed as a potential conflict of interest.

Publisher's Note: All claims expressed in this article are solely those of the authors and do not necessarily represent those of their affiliated organizations, or those of the publisher, the editors, and the reviewers. Any product that may be evaluated in this article, or claim that may be made by its manufacturer, is not guaranteed or endorsed by the publisher.

Copyright (C) 2022 Tan, Ke, Li, Chen, Zheng, Wang, Zhou, Huang and Li. This is an open-access article distributed under the terms of the Creative Commons Attribution License (CC BY). The use, distribution or reproduction in other forums is permitted, provided the original author(s) and the copyright owner(s) are credited and that the original publication in this journal is cited, in accordance with accepted academic practice. No use, distribution or reproduction is permitted which does not comply with these terms. 